\title{
Anxiety affects the amplitudes of red and green color- elicited flash visual evoked potentials in humans
}

\author{
Yuki Hosono", ${ }^{1,2}$ Kazuyoshi Kitaoka ${ }^{3}$, Ryo Urushihara ${ }^{4}$, Hiroyoshi Séi', and \\ Yohsuke Kinouchi ${ }^{5}$ \\ ${ }^{1}$ Department of Integrative Physiology, Institute of Health Biosciences, the University of Tokushima \\ Graduate School, Tokushima, Japan, ${ }^{2}$ Ritsumeikan University Institute for General Education, Kyoto, \\ Japan, ${ }^{3}$ Department of Physiology, Institute of Health Biosciences, the University of Tokushima Gradu- \\ ate School, Tokushima, Japan, ${ }^{4}$ Ritsumeikan University College of Social Sciences, Kyoto, Japan, ${ }^{5}$ De- \\ partment of Electrical and Electronic Engineering, Institute of Technology and Science, the University \\ of Tokushima Graduate School, Tokushima, Japan
}

\begin{abstract}
It has been reported that negative emotional changes and conditions affect the visual faculties of humans at the neural level. On the other hand, the effects of emotion on color perception in particular, which are based on evoked potentials, are unknown. In the present study, we investigated whether different anxiety levels affect the color information processing for each of 3 wavelengths by using flash visual evoked potentials (FVEPs) and State-Trait Anxiety Inventory. In results, significant positive correlations were observed between FVEP amplitudes and state or trait anxiety scores in the long (sensed as red) and middle (sensed as green) wavelengths. On the other hand, short-wavelength-evoked FVEPs were not correlated with anxiety level. Our results suggest that negative emotional conditions may affect color sense processing in humans. J. Med. Invest. 61 : 79-83, February, 2014
\end{abstract}

Keywords : flash visual evoked potentials, light emission diodes, color perception, State-Trait Anxiety Inventory

\section{INTRODUCTION}

It is widely reported that negative emotional changes and conditions affect the visual faculties of humans at the neural level. Numerous reports have shown that the contrast gain of pattern electroretinograms was impaired in patients with major depression (1-3). It has also been suggested that negative emotional states, such as depression and anxiety, affect visual evoked potentials (VEPs) $(4,5)$ and visual

Received for publication November 5, 2013 ; accepted November 30, 2013.

Address correspondence and reprint requests to Kazuyoshi Kitaoka, Ph.D., Department of Physiology, Institute of Health Biosciences, the University of Tokushima Graduate School, 3-1815, Kuramoto-cho, Tokushima 770-8503, Japan and Fax : +8188-633-9443. event-related potentials $(6,7)$. Fitzgerald described this phenomenon as such individuals having "graycolored glasses" (8). On the other hand, emotional effects on color perception are largely unknown. Trichromatic primates including humans have 3 types of cone photoreceptors, which are classed into L (long), M (middle), and S (short) categories because of the wavelength band they are sensitive to in the visible spectrum. Excitation of these photoreceptors is responsible for color vision of red, green, and blue respectively $(9,10)$. It is possible that the emotional effects on the visual functions have colorspecificity because the color information from these photoreceptors project to primary visual cortex (V1) selectively (9). At the behavioral level, Wu et al. reported that depressive tendencies correlated with 
the color preference used on self-portraits (11). However, the study was not, to the best of our knowledge, based on any neural activity. In the present study, we investigated whether the difference in anxiety levels affects the visual information processing for each of the 3 wavelengths by using flash visual evoked potentials (FVEPs) and State-Trait Anxiety Inventory (STAI).

\section{METHODS}

Seventeen male individuals with normal vision participated in this study (mean age \pm SD : $20.7 \pm$ 2.3 years). All subjects participated in the study were free from neurological and psychiatric diseases with no known abnormalities of the visual system. All participants signed informed consent prior to participating in the study. This study was approved by the ethics committee of Tokushima University Hospital (No. 1142) and conformed to the principles outlined in the latest revision of the Declaration of Helsinki.

Before FVEP recording, Participants completed the Japanese version of the STAI (Jitsumukyoikushuppan, Tokyo, Japan) to estimate their anxiety levels. The results of the STAI were a state and trait anxiety score. After this, the participants were prepared for electroencephalograph (EEG) recording and seated comfortably in an electrically shielded and darkened room. They were instructed to fixate on the center of a light emitting diode (LED) stimulator that was placed on $100 \mathrm{~cm}$ before them. The stimulator was capable of producing 3 wavelengths : (470 nm (blue), $527 \mathrm{~nm}$ (green), and $635 \mathrm{~nm}$ (red) ; NSSM065, Nichia Corp., Tokushima, Japan). Flashlights in each wavelength from the LED (intensity : $2 \mathrm{~cd} / \mathrm{m}^{2}$, duration : $300 \mathrm{~ms}$, interstimulus interval : $1200-1600 \mathrm{~ms}$, in a pseudorandom order) were presented separately in each wavelength condition. The order of stimulus conditions was randomized for each participant. The EEGs were recorded on an apparatus that consisted of silver chloride disk surface electrodes at $\mathrm{Fz}, \mathrm{Cz}, \mathrm{Pz}$, and $\mathrm{Oz}$, in accordance with the International 10-20 system. Electrode impedance was kept below $3 \mathrm{k} \Omega$. An electrooculogram (EOG) was also recorded with a pair of silver chloride disk electrodes at $2 \mathrm{~cm}$ above and $2 \mathrm{~cm}$ below the right outer canthus. The linked earlobe was used for reference. The ground electrode was placed at Fpz. The EEG and EOG signals were amplified and acquired at a sampling rate of $10 \mathrm{kHz}$ and filtered at $1-5000$ and $0.5-1000 \mathrm{~Hz}$, respectively by SCAN 4.1 software (Neuro Scan Labs, Charlotte, NC). The FVEPs were averaged from 100 stimuli in each wavelength condition from $30 \mathrm{~ms}$ before to $300 \mathrm{~ms}$ after the LED stimulus onset in offline analysis. The artifacts were eliminated by referring to the EOG data. Analyzed components which were detected in all participates consisted of negative peaks with a latency of 65-95 ms (N2) and about $130 \mathrm{~ms}(\mathrm{~N} 3)$, and positive peaks with a latency of about $120 \mathrm{~ms}$ (P2) and 140-160 ms (P3). The waveform components of FVEPs were identified in accordance with the standards of the International Society for Clinical Electrophysiology of Vision (ISCEV) (12). The baselineto-peak amplitudes of these components were measured. The baseline was defined as the segment between 2 and $6 \mathrm{~ms}$ after stimulation.

Comparison between the values of each component of the waveform in each stimulus conditions were analyzed by an ANOVA and Scheffe's post-hoc test. Correlations between state and trait anxiety scores and FVEP components were evaluated by analysis of their Pearson correlation coefficients. All data were analyzed with SPSS version $11.01 \mathrm{~J}$ for Windows (SPSS Japan Institute Inc., Tokyo). $\mathrm{P}<$ 0.05 was considered statistically significant.

\section{RESULTS}

Grand average FVEP waveforms for each electrode and wavelengths are shown in Figure 1. The latency of each peak was not significantly different. On the other hand, a one-way ANOVA demonstrated a significant difference in the amplitudes of FVEPs in $\mathrm{Fz}[\mathrm{N} 2: \mathrm{F}(2,48)=4.329, \mathrm{P}<0.05, \mathrm{P} 3: \mathrm{F}$ $(2,48)=8.220, \mathrm{P}<0.01], \mathrm{Cz}[\mathrm{N} 2: \mathrm{F}(2.48)=7.866, \mathrm{P}<$ $0.01, \mathrm{P} 2: \mathrm{F}(2,48)=4.118, \mathrm{P}<0.05, \mathrm{~N} 3: \mathrm{F}(2,48)=$ 4.985, $\mathrm{P}<0.05, \mathrm{P} 3: \mathrm{F}(2,48)=8.385, \mathrm{P}<0.01], \mathrm{Pz}$ $[\mathrm{N} 2: \mathrm{F}(2.48)=8.968, \mathrm{P}<0.01, \mathrm{P} 2: \mathrm{F}(2,48)=3.453$, $\mathrm{P}<0.05, \mathrm{P} 3: \mathrm{F}(2,48)=7.147, \mathrm{P}<0.01]$, and $\mathrm{O} z[\mathrm{~N} 2$ : $\mathrm{F}(2.48)=4.985, \mathrm{P}<0.05]$. The post hoc test clarified that the short-wavelength (blue)-evoked FVEPs showed larger amplitudes than long (red) - or middle (green)-wavelength-evoked FVEPs (Figure 1). Correlation coefficients between the amplitudes of FVEP obtained at each wavelength and state and trait anxiety scores estimated by the STAI are indicated in Table 1 . In state anxiety, a significant positive correlation was observed with the amplitude of the P3 component of FVEPs evoked in green light from the $\mathrm{Fz}$ region $(\mathrm{R}=0.482, \mathrm{P}<0.05)$. In trait anxiety, a 
significant correlation was observed with the amplitude of the N3 component of FVEPs evoked in red light-evoked from the $\mathrm{C} z$ region $(\mathrm{R}=0.495, \mathrm{P}<0.05)$.
Other peaks and region were not significantly correlated. These data plots are shown in Figure 2.

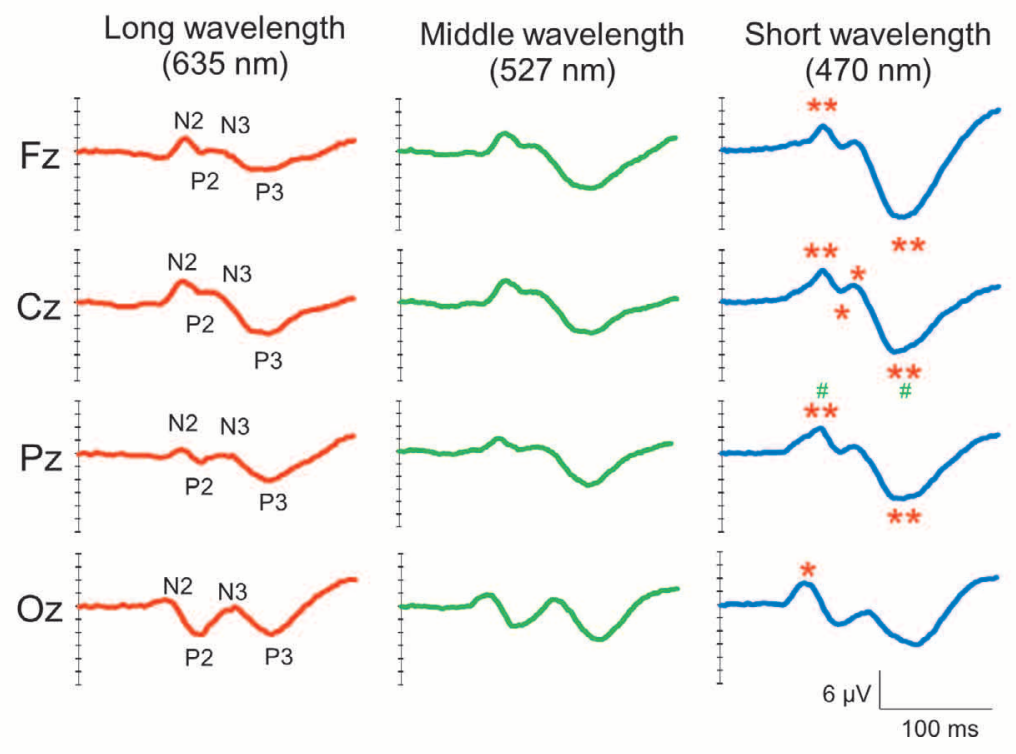

Figure 1. Grand average FVEP waveforms from all electrodes and wavelengths in 17 participants. Short-wavelength-evoked FVEPs show greater amplitudes compared with long- and middle-wavelength FVEPs. Latency did not change significantly. ${ }^{*} \mathrm{P}<0.05$, ${ }^{* \star} \mathrm{P}<0.01$ vs long wavelengths, $\# \mathrm{P}<0.05$ vs middle wavelengths.

Table 1. Coefficient correlation between FVEP amplitudes in each wavelength and STAI scores.

\begin{tabular}{|c|c|c|c|c|c|c|c|c|c|c|c|}
\hline & & $\begin{array}{l}\text { STAI } \\
\text { score }\end{array}$ & $\begin{array}{c}\text { long } \\
(635 \mathrm{~nm})\end{array}$ & $\begin{array}{c}\text { middle } \\
(527 \mathrm{~nm})\end{array}$ & $\begin{array}{c}\text { short } \\
(470 \mathrm{~nm})\end{array}$ & & & $\begin{array}{l}\text { STAI } \\
\text { score }\end{array}$ & $\begin{array}{c}\text { long } \\
(635 \mathrm{~nm})\end{array}$ & $\begin{array}{c}\text { middle } \\
(527 \mathrm{~nm})\end{array}$ & $\begin{array}{c}\text { short } \\
(470 \mathrm{~nm})\end{array}$ \\
\hline \multirow{9}{*}{$\mathrm{Fz}$} & \multirow{2}{*}{$\mathrm{N} 2$} & state & 0.354 & 0.197 & 0.162 & \multirow{2}{*}{\multicolumn{2}{|c|}{$\mathrm{N} 2$}} & state & 0.055 & 0.092 & -0.091 \\
\hline & & trait & 0.231 & 0.103 & -0.036 & & & trait & -0.088 & 0.016 & -0.268 \\
\hline & \multirow{2}{*}{$\mathrm{P} 2$} & state & 0.41 & 0.444 & 0.462 & \multirow{5}{*}{$\mathrm{Pz}$} & \multirow{2}{*}{ P2 } & state & 0.215 & 0.062 & 0.275 \\
\hline & & trait & 0.31 & 0.238 & 0.286 & & & trait & 0.072 & -0.092 & 0.122 \\
\hline & & & & & & & & & & & \\
\hline & \multirow{2}{*}{ N3 } & state & 0.33 & 0.262 & 0.087 & & \multirow{2}{*}{ N3 } & state & 0.139 & -0.187 & -0.234 \\
\hline & & trait & 0.334 & 0.042 & -0.039 & & & trait & 0.202 & -0.201 & -0.373 \\
\hline & \multirow{2}{*}{ P3 } & state & 0.179 & $0.482 *$ & 0.37 & & \multirow{2}{*}{ P3 } & state & 0.085 & -0.302 & 0.116 \\
\hline & & trait & 0.178 & 0.219 & 0.19 & & & trait & 0.033 & -0.466 & 0.115 \\
\hline \multirow{9}{*}{$\mathrm{C} z$} & \multirow{2}{*}{$\mathrm{N} 2$} & state & 0.265 & 0.198 & 0.004 & \multirow{9}{*}{$\mathrm{O} z$} & \multirow{2}{*}{$\mathrm{N} 2$} & state & -0.294 & -0.241 & -0.006 \\
\hline & & trait & 0.148 & 0.122 & -0.128 & & & trait & -0.257 & -0.042 & -0.042 \\
\hline & \multirow{2}{*}{$\mathrm{P} 2$} & state & 0.351 & 0.318 & 0.328 & & \multirow{2}{*}{ P2 } & state & -0.073 & 0.016 & 0.117 \\
\hline & & trait & 0.27 & 0.179 & 0.24 & & & trait & -0.173 & -0.025 & -0.025 \\
\hline & & & & & & & & & & & \\
\hline & \multirow{2}{*}{ N3 } & state & 0.446 & 0.052 & -0.187 & & \multirow{2}{*}{ N3 } & state & -0.218 & -0.288 & -0.362 \\
\hline & & trait & $0.495^{*}$ & -0.062 & -0.237 & & & trait & -0.173 & -0.359 & -0.359 \\
\hline & \multirow{2}{*}{ P3 } & state & 0.197 & 0.104 & 0.332 & & \multirow{2}{*}{ P3 } & state & 0.127 & -0.313 & -0.393 \\
\hline & & trait & 0.177 & -0.103 & 0.251 & & & trait & 0.021 & -0.421 & -0.421 \\
\hline
\end{tabular}


A

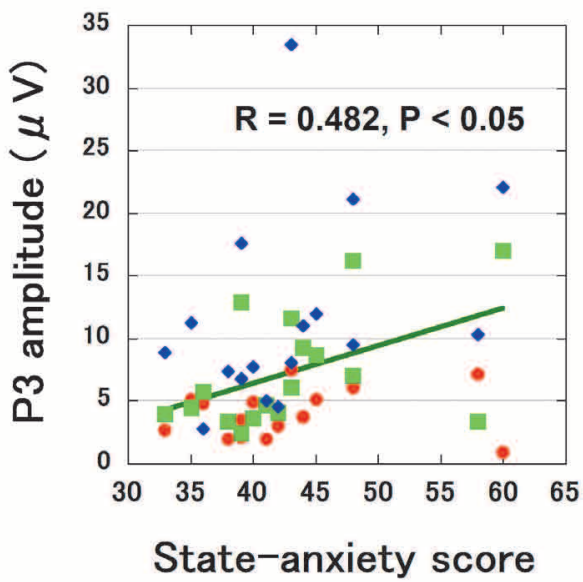

B

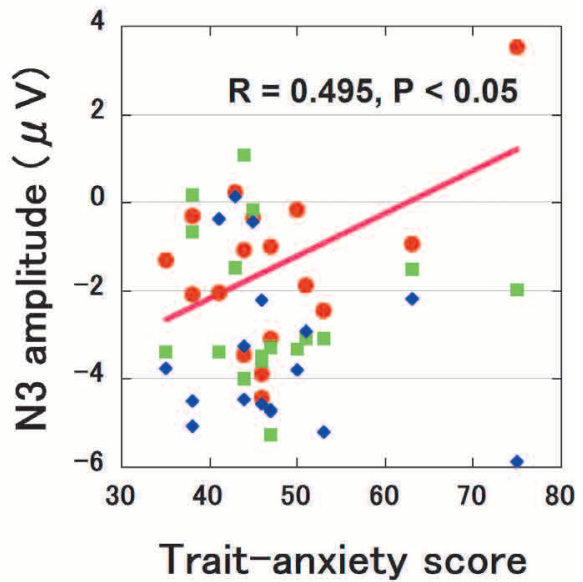

Figure 2. Coefficient correlations between state anxiety score and P3 amplitude from $\mathrm{Fz}(\mathrm{A})$ and trait anxiety score and N3 amplitude from $\mathrm{C} z$ (B). Each data point from short-, middle-, and long-wavelength-evoked FVEPs are shown in blue, green, and red plots, respectively. Significant positive correlations were observed in the middle and long wavelengths. Short-wavelength-evoked FVEPs did not show significant correlation with any amplitude.

\section{DISCUSSION}

The present results suggest that the processing of spectral information has some specificity, and negative emotional conditions affected this processing in the middle and long wavelengths studied.

In grand averaged FVEPs, the short-wavelengthevoked FVEPs showed larger amplitudes than longand middle-wavelength-evoked FVEPs in spite of equal LED intensity. Rodents and primates have Melanopsin-containing intrinsically photosensitive retinal ganglion cells (ipRGCs) that facilitate circadian photoentrainment (13). However, recent reports suggest that the ipRGCs also contribute to image-forming vision (14). Due to the peak sensitivity of Melanopsin being around 470-480 nm in humans (13), the enlarged amplitudes in short-wavelength-evoked FVEPs in our study may be a result of the contribution of both S cone cells and ipRGCs. On the other hand, the peak sensitivity of rod cell is around $490 \mathrm{~nm}(10)$, so the possibility that the excitation of rod cells affects the amplitudes in shortwavelength-evoked FVEPs also needs to be considered. Further study will be needed to clarify the mechanisms of these amplitude differences.

With regard to the correlations between FVEPs and STAI scores, the amplitudes of P3 in middlewavelength-evoked FVEPs from Fz and N3 in longwavelength-evoked FVEPs from $\mathrm{C} z$ showed significant positive correlations with state and trait anxiety scores, respectively. These results suggest that anxiety affects secondary visual processing in prefrontal regions, after V1. Similarly to our study, Shackman et al. reported that the amplitude of early components (184-236 ms, N1) of event related potentials in prefrontal regions evoked by a visual discrimination task was increased by threatening stress (7). They suggested that the stress-induced amplification of N1 stems from modulation of extrastriate cortex by the amygdala or lateral prefrontal cortex. The anxiety-induced amplification of N3 and P3 in our FVEPs may be also derived from a modulation of extrastriate cortex. However, short-wavelength-evoked FVEPs did not show any significant correlation with STAI scores. Color information from each class (S, $\mathrm{M}$, and $\mathrm{L}$ ) of cone photoreceptors project V1 via the parvocellular layers of the lateral geniculate nucleus. In this pathway, color information converts to 2 sets of the color-opponency system : red-green (represented by L-on/M-off and M-on/L-off) and blue-yellow (represented by S-on/ $(\mathrm{L}+\mathrm{M})$-off and $(\mathrm{L}+\mathrm{M})$-on/S-off) (9). These differences in the color-projecting pathway may be involved with colorspecificity in the FVEP-anxiety correlation uncovered in this study. In addition, Melanopsin-derived input from ipRGCs may also be related to this difference.

Many early studies reported that negative emotional changes deteriorate contrast perception (1-4). Similarly, the long and middle wavelengths, which were affected by anxiety level in our study, are one of the color-opponency systems that contribute to red-green color contrast. Our present study indicated the possibility that perception of color contrast 
is affected by negative emotion. The alteration of color contrast perception might also contribute to the "gray-colored glass" of depressive and anxious individuals, and not only changes in their contrast perception.

\section{DISCLOSURE}

All of the authors, Yuki Hosono, Kazuyoshi Kitaoka, Ryo Urushihara, Hiroyoshi Séi, and Yohsuke Kinouchi, declare that they have no conflict of interest or financial ties to disclose.

\section{ACKNOWLEDGEMENTS}

This work was supported by Management Expenses Grants for National University Corporations, The Ministry of Education, Culture, Sports, Science and Technology (MEXT). We thank Ryuji Kaji (Department of Neurology, the University of Tokushima Graduate School) and Masaki Hashizume (Institute of Technology and Science, the University of Tokushima Graduate School) for great assistance in carrying out this study.

\section{REFERENCES}

1. Bubl E, Kern E, Ebert D, Bach M, Tebartz van Elst L: Seeing gray when feeling blue? Depression can be measured in the eye of the diseased. Biol Psychiatry 68 : 205-208, 2010

2. Bubl E, Ebert D, Kern E, Tebartz van Elst L, Bach $\mathrm{M}$ : Effect of antidepressive therapy on retinal contrast processing in depressive disorder. Br J Psychiatry 201 : 151-158, 2012

3. Fam J, Rush AJ, Haaland B, Barbier S, Luu C : Visual contrast sensitivity in major depressive disorder. J Psychosom Res 75 : 83-86, 2013

4. Laretzaki G, Plainis S, Argyropoulos S, Pallikaris
IG, Bitsios P : Threat and anxiety affect visual contrast perception. J Psychopharmacol 24 : 667-675, 2010

5. Normann C, Schmitz D, Furmaier A, Doing $\mathrm{C}$, Bach $\mathrm{M}$ : Long-term plasticity of visually evoked potentials in humans is altered in major depression. Biol Psychiatry $62: 373-380,2007$

6. Weinstein AM : Visual ERPs evidence for enhanced processing of threatening information in anxious university students. Biol Psychiatry $37: 847-858,1995$

7. Shackman AJ, Maxwell JS, McMenamin BW, Greischar LL, Davidson RJ : Stress potentiates early and attenuates late stages of visual processing. J Neurosci 31 : 1156-1161, 2011

8. Fitzgerald PJ : Gray colored glasses : Is major depression partially a sensory perceptual disorder? J Affect Disord 151 : 418-422, 2013

9. Conway BR, Chatterjee S, Field GD, Horwitz GD, Johnson EN, Koida K, Mancuso K: Advances in color science : from retina to behavior. J Neurosci 30 : 14955-14963, 2010

10. Schnapf JL, Kraft TW, Nunn BJ, Baylor DA : Spectral sensitivity of primate photoreceptors. Vis Neurosci $1: 255-261,1988$

11. Wu F, Chang E, Lee Y : Manifestation of depressive tendency in color perception and colors utilized in creating a self-portrait. Color Res Appl 34 : 84-92, 2009

12. Odom JV, Bach M, Brigell M, Holder GE, McCulloch DL, Tormene AP, Vaegan : ISCEV standard for clinical visual evoked potentials (2009 update). Doc Ophthalmol 120 : 111-119, 2010

13. Hankins MW, Peirson SN, Foster RG : Melanopsin : an exciting photopigment. Trends Neurosci $31: 27-36,2008$

14. Schmidt TM, Do MT, Dacey D, Lucas R, Hattar S, Matynia A : Melanopsin-positive intrinsically photosensitive retinal ganglion cells : from form to function. J Neurosci 31 : 16094-16101, 2011 